\title{
Um programa de atividade física generalizada leva a manutenção funcional em indivíduos com doença de Parkinson
}

http://dx.doi.org/10.11606/1807-5509201900040611

\section{Resumo}

A Doença de Parkinson (DP) é uma doença crônico-neurodegenerativa, caracterizada principalmente por: tremor de repouso, rigidez, bradicinesia, alterações da marcha e instabilidade postural. Com isso, há uma diminuição na qualidade de vida e na independência dos idosos com DP, com redução na habilidade de desempenhar atividades da vida diária. Assim, diversas propostas têm sido feitas no intuito de auxiliar o tratamento medicamentoso, como a pratica de atividade física. Entretanto, a maior parte dessas propostas envolve programas de pequena duração ou que foquem em pontos específicos da doença. Dessa forma, o principal objetivo do presente estudo foi avaliar o efeito de um treinamento multimodal de 8 meses sobre o equilibrio dinâmico e a mobilidade funcional de pacientes com Doença de Parkinson idiopática, por meio da Escala de Equilibrio Funcional de Berg (EEFB) e do teste Timed "Up and Go" (TUG). Participaram do estudo 8 voluntários nos estágios de leve à moderado da doença (Estágios de Hoehn e Yahr = 1 a 3), cognitivamente preservados, avaliados em sob efeito da medicação, antes e após a realização do protocolo de exercícios proposto. Também foram avaliados após 3 meses do término do treinamento, com intuito de avaliar o efeito do destreino nesses indivíduos e não foram encontradas alterações na EEFB e TUG. Dessa forma, podemos afirmar que mesmo com o avanço da DP, esse programa de treinamento foi eficaz na manutenção do equilíbrio dinâmico e na mobilidade funcional desses sujeitos por meio da atividade física.

Palavras-Chave: Mobilidade Funcional; Equilíbrio Dinâmico; Envelhecimento; Exercício Físico; Avaliação Clínica.

\section{Introdução}

A doença de Parkinson (DP) é caracterizada como uma patologia neurodegenerativa e idiopática, que acomete de 0,5 a $1 \%$ da população entre 65 e 69 anos $^{1}$, atingindo cerca de 3,3\% da população brasileira acima de 64 anos $^{2}$. A DP é desencadeada pela degeneração progressiva de neurônios dopaminérgicos da substância negra parte compacta, que são produtores da dopamina ${ }^{3}$, sendo esse neurotransmissor um regulador da atividade motora, entre outras funçôes ${ }^{4}$. As alteraçôes patológicas nos núcleos da base presentes na DP levam aos seus principais sintomas: tremor em repouso, rigidez muscular, bradicinesia, alteraçōes na marcha e instabilidade postural ${ }^{5,6}$. Alguns sintomas da DP são acentuados durante a realização de sequências de movimentos bem aprendidos e automáticos, tais como caminhar e durante a mudança de um movimento para outro ${ }^{7}$. Estes distúrbios afetam principalmente a realização de movimentos repetitivos, simultâneos e sequenciais e induzem alteraçôes no controle do equilíbrio ${ }^{8}$, sendo essas alterações, instabilidade postural e déficits na marcha, potenciais fatores de risco de quedas em pacientes com $\mathrm{DP}^{9}$.

O principal tratamento para a DP é a terapia medicamentosa e há evidências de que a medicação antiparkinsoniana, sobretudo a levodopa (metabólico precursor da dopamina), não reduz problemas no equilíbrio e em deficiências da marcha, limitado também por um efeito teto com a progressão da
*School of Public Healthy and Community Medicine, University of New South Wales, Sydney, Austrália. ${ }^{* *}$ Neuroscience Research Australia, Falls, Balance and Injury Research Centre, Sydney, Austrália.

***Universidade Estadual Paulista, Campus de Rio Claro, Rio Claro, Brasil.

${ }^{* * * *}$ Departamento de Esportes, Universidade Federal do Espirito Santo, Vitória, Brasil. 
doença e esse efeito resulta em periodos menores de tempo efetivo para alivio dos sintomas ${ }^{10}$. Essa melhora de alguns sintomas sem melhora no equilíbrio dinâmico pode resultar em um paradigma perigoso ao paciente, tendo estes a sua mobilidade aumentada, porém, ficam mais susceptíveis à quedas e sem melhoras quanto às respostas posturais ${ }^{11}$. Como coadjuvante a essa terapia medicamentosa, programas de exercício físico têm sido considerados estratégias eficientes para melhorar as funções físicas, a força, o equilíbrio, a marcha e a qualidade de vida ${ }^{12}$. O exercício é uma intervenção não-farmacológica economicamente viável, sustentável e terapêutica com pouco ou nenhum risco e com significativos benefícios para a saúde ${ }^{12}$. Essa proposta tem sido feita, pois além dos idosos com DP apresentarem os sintomas característicos da doença, apresentam também alterações músculo-esqueléticas como redução da força, diminuição da flexibilidade, distúrbios no equilíbrio e consequentemente quedas ${ }^{13,14}$. Assim, esses indivíduos apresentam um nível de atividade física 15\% inferior comparados a idosos saudáveis, na mesma faixa etária ${ }^{15}$ interferindo diretamente na performance funcional e independência destes indivíduos.

GobBi, et al. ${ }^{16}$ e ViTÓrio, et al. ${ }^{17}$ encontraram interessantes resultados, como melhoras no equilíbrio, mobilidade funcional e parâmetros do andar após um período de 6 meses de intervenção multimodal em pacientes com DP. Entretanto, essas melhoras não foram acompanhadas após o fim do período de intervenção $($ follow-up). A literatura tem trazido que mesmo após exercícios básicos, dança ou até mesmo após programas de atividade física generalizada para idosos com DP, esses indivíduos têm demonstrado melhoras e manutenção nos componentes da capacidade funcional, mobilidade funcional e nas variáveis temporais e espaço-temporais da marcha ${ }^{18,15}$. Esses estudos demonstraram que os efeitos induzidos pelo treinamento permanecem por períodos de 4 semanas à 6 meses após a interrupção dos exercícios, mesmo sendo exercícios com características bem distintas entre eles (exercícios generalizados e dança).
Esses resultados são importantes, pois demonstram que a prática de atividade física não apenas melhora pontualmente a qualidade de vida desses indivíduos, mas demonstra efeito por períodos de médio prazo.

Devido ao conhecimento de que várias modalidades de exercícios físicos têm apresentado melhoras de resultados funcionais em indivíduos com DP, o exercício multimodal tem sido proposto, pois trabalha com todos os componentes da capacidade funcional dos indivíduos, e executado com uma progressão e dificuldade acrescida ao decorrer do programa, proporciona melhoras globais ao indivíduo com DP. Dessa forma, um dos objetivos do presente estudo foi realizar um programa de atividade multimodal que englobasse várias modalidades de exercícios buscando além de trabalhar todos os componentes da capacidade funcional, trazer melhora na qualidade de vida desses indivíduos. Isso foi esperado, pois o programa proposto envolveu atividades em grupo, motivando os participantes, com atividades diversificadas, levando a uma grande aderência, proporcionando bem estar aos mesmos. Além disso, esperamos que um programa de exercício multimodal com maior duração (8 meses) pudesse ser tão efetivo quanto um programa multimodal com duração de apenas 6 meses ${ }^{16,17}$. Além disso, um maior tempo de atividade física oferecido aos participantes, representa um maior tempo de convívio social, e outros benefícios nessa esfera podem ser alcançados. Outros objetivos do estudo foram avaliar o efeito do mesmo programa no equilíbrio dinâmico, mobilidade funcional e nas variáveis clínicas de idosos com DP idiopática, assim como realizar a avaliação do destreino com intuito de verificar se os efeitos do treinamento se mantiveram após um período de três meses. A hipótese do presente estudo é de que um programa multimodal de longa duração, mesmo que seja um programa piloto, irá trazer melhoras ou manutenção funcional para pacientes com DP imediatamente após um período de intervenção. Ainda, acreditamos na manutenção desses efeitos após um período de três meses sem atividade física regular.

\section{Método}

O estudo foi previamente aprovado pelo Comitê de Ética do Instituto de Biociências da Universidade Estadual Paulista "Júlio de Mesquita Filho", campus Rio Claro (176/2007), e após a leitura e aceite por parte dos participantes, o estudo foi conduzido. Uma amostra por conveniência foi determinada para esse estudo. Por se tratar de um estudo piloto, com intenção de verificar a eficácia de um 
treinamento multimodal por um período prolongado (8 meses) e por termos poucos pacientes com DP que se voluntariaram a participarem desse estudo, preferimos adotar somente um único grupo de intervenção nesse presente estudo. Participaram do estudo 8 pacientes com DP idiopática (cinco do sexo masculino), com idade de 71,13 \pm 7,98 anos, com presença de DP há $8 \pm 5,74$ anos, massa corporal de $62,74 \pm 11,1 \mathrm{~kg}$, estatura de $161,51 \pm 9,67 \mathrm{~cm}$, e preservados cognitivamente. Os pacientes foram avaliados sob efeito da medicação antiparkinsoniana, assim como realizaram as atividades sob efeito medicamentoso. As avaliaçōes realizadas foram antes (Pré), imediatamente após o treinamento (Pós) e 3 meses após seu termino (destreino - DT).

Os pacientes foram submetidos as avaliações:

- UPDRS para avaliar a condição clínica do paciente. Foram avaliados a condição clínica funcional (UPDRSII) e motora (UPDRSIII), assim como o comprometimento total ocasionado pela $\mathrm{DP}^{19}$;

- Escala de Hoehn e Yahr (HY) com a finalidade de identificar os estágios da $\mathrm{DP}^{20}$;

- Escala de Equilíbrio Funcional de Berg (EEFB), para avaliar o equilíbrio funcional dos pacientes. Tal escala é composta por 14 itens pontuados entre 0 (pior desempenho) e 4 (melhor desempenho) envolvendo tarefas funcionais específicas em diferentes bases de apoio ${ }^{21}$;

- $\quad$ Timed Up and Go (TUG) para avaliar a mobilidade functional básica. Para realizar o teste o participante levanta de uma cadeira a partir da posição sentada, percorre uma distância de 3 metros, contorna um obstáculo e senta novamente, realizando a tarefa o mais rápido possível. O tempo média de 3 tentativas é contabilizado ${ }^{22}$.

O Programa de treinamento foi multimodal visando melhora na capacidade funcional, sendo que altos níveis de força, flexibilidade e mobilidade levam a melhoras na qualidade de vida e realização das AVDs ${ }^{23-25}$ e foi composto por 5 componentes que são: força muscular, equilíbrio corporal, coordenação motora, resistência aeróbia e flexibilidade. O treinamento teve duração de 8 meses (96 sessões), realizado 3 vezes por semana, com sessōes de 60 minutos. Ele foi dividido em 2 períodos de 4 meses cada, sendo cada período subdividido em 3 fases, sendo cada fase composta por 4 ciclos e cada ciclo por 4 sessões e iniciado após as avaliações iniciais. Essas sessões foram enumeradas entre 1 a 4 , e cada uma conteve:

- Sessão 1: alongamento inicial (10'), trabalho de coordenação motora $\left(20^{\prime}\right)$, trabalho de fortalecimento muscular (20') e alongamento final $\left(10^{\prime}\right)$;

- Sessão 2: alongamento inicial (10'), trabalho de fortalecimento muscular (20'), trabalho de resistência aeróbia associado à coordenação motora $\left(20^{\prime}\right)$ e alongamento final (10');

- Sessão 3: alongamento inicial (10'), trabalho de resistência aeróbia $\left(25^{\prime}\right)$ e trabalho de flexibilidade $\left(25^{\prime}\right)$;

- Sessão 4: alongamento inicial (10'), trabalho de equilíbrio corporal (20'), trabalho de coordenação motora associado à resistência aeróbia (20') e alongamento final (10').

Esse programa de treinamento teve progressão de acordo com seus componentes que foram:

- Força muscular: a cada 2 ciclos ocorreu o aumento do número de séries ou repetiçōes, e a cada fase ocorreu o aumento da carga;

- $\quad$ Equilíbrio corporal: a cada 2 ciclos ocorreu o aumento da complexidade (por exemplo, diminuição da base de suporte, e posteriormente, trabalho com equilíbrio unipodal) e a cada fase teve combinação com diferentes perturbações posturais (por exemplo, olhos fechados, e posteriormente uso de superfície mais instável);

- Coordenação motora: a cada ciclo ocorreu a variação de materiais utilizados e a cada fase ocorreu o aumento da complexidade (por exemplo, atividade de coordenação motora, em uma velocidade elevada);

- Resistência aeróbia: a cada 2 ciclos ocorreram o aumento do número de séries e a diminuição do tempo de recuperação, e a cada fase houve o aumento da intensidade e complexidade do ambiente;

- Flexibilidade: a cada ciclo ocorreu a variação da articulação trabalhada e a cada fase as séries aumentaram e houve a variação dos materiais complementares.

Terminando o primeiro período, os participantes deram continuidade no treinamento e seguiram o mesmo modelo, com as mesmas fases, ciclos e sessões, inclusive com a mesma forma de progressão.

Para análise estatística, vimos que após a verificação de normalidade dos dados por meio do teste de Shapiro-Wilk, a influência do treinamento sobre todas as variáveis (exceto para TUG e UPDRS III) foi avaliada por meio de uma Análise de Variância one-way para medidas repetidas considerando o período como fator (Pré x Pós x DT). Já a influência do período sobre os valores de TUG e UPDRS III foi avaliada por meio do Teste de Friedman. O nível de significância foi estabelecido em 0,05 e todos os testes foram realizados no software Statistica ${ }^{\circledR} 7.0$. 


\section{Resultados}

Na TABELA 1, são descritos os resultados clínicos dos participantes em cada período de avaliação.

Pode-se observar na TABELA 1 que não houve diferença estatística significativa em nenhum dos períodos de treinamento.

$\mathrm{Na}$ FIGURA 1 observamos que não houve diferente estatística significativa após o período de intervenção. Porém observamos manutenção dos valores do tempo despendido para a realização do TUG em Pré em relação a Pós, e essa manutenção se mantém no DT (Pré=10,73 \pm 7,61 segundos; Pós=10,08 \pm 5,92 segundos; $\mathrm{DT}=9,35 \pm 5,11 ; \mathrm{p}=0,284)$.

TABELA 1-Valores médios e desvio padrão das variáveis clínicas dos participantes: Pré (antes do programa de treinamento), Pós (após o programa de treinamento), DT (destreino).

\begin{tabular}{ccccc}
\hline & Pré & Pós & DT & p \\
\hline UPDRSII (Pontos) & $14 \pm 4,42$ & $13,25 \pm 3,83$ & $13,63 \pm 4,72$ & 0,94 \\
UPDRSIII (Pontos) & $29,13 \pm 12,82$ & $26,13 \pm 11,89$ & $28,63 \pm 13,20$ & 0,28 \\
UPDRS Total (Pontos) & $46,25 \pm 15,56$ & $42,13 \pm 13,50$ & $45,50 \pm 17,44$ & 0,90 \\
HY (Estágio) & $1,56 \pm 0,73$ & $1,81 \pm 0,61$ & $1,88 \pm 0,60$ & 0,64 \\
\hline
\end{tabular}
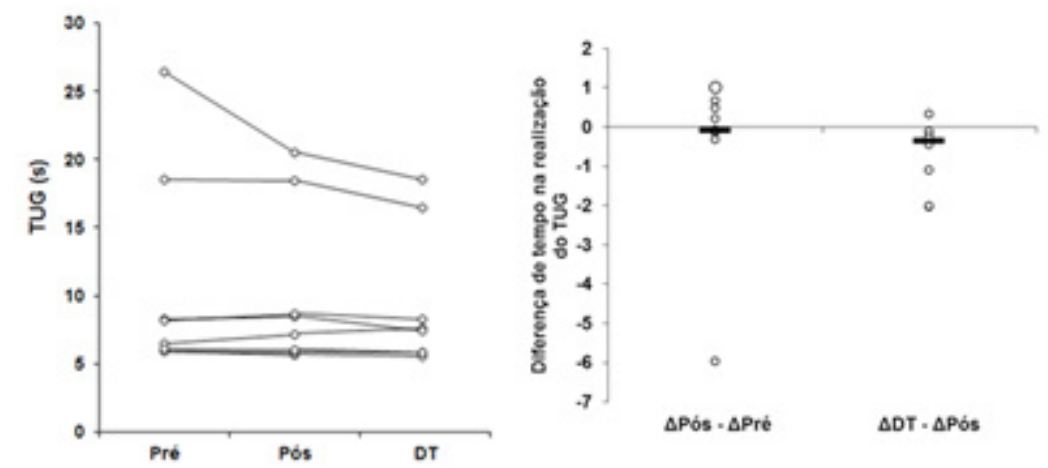

FIGURA 1 -Valores médios do TUG, Pré (antes do programa de treinamento), Pós (após o programa de treinamento) e DT (destreino) e diferença entre os períodos de intervenção.

A FIGURA 2 representa os valores médios e desvios padrão da EEFB, onde os valores se mantiveram similares nas três condiçóes (Pré $=52,13 \pm 3,8$ pontos; Pós $=52,5 \pm 4,69$ pontos; DT $=51,63 \pm 4$ pontos), não havendo diferença estatística significativa $(\mathrm{p}=0,91)$.
Por fim, observamos na FIGURA 3 que representa os valores médios e desvios padrão da escala de HY (Pré=1,56 $\pm 0,78$; Pós $=1,81 \pm$ $0,65 ; \mathrm{DT}=1,88 \pm 0,64 ; \mathrm{p}=0,64)$, que não houve progressão estatisticamente significante da DP.
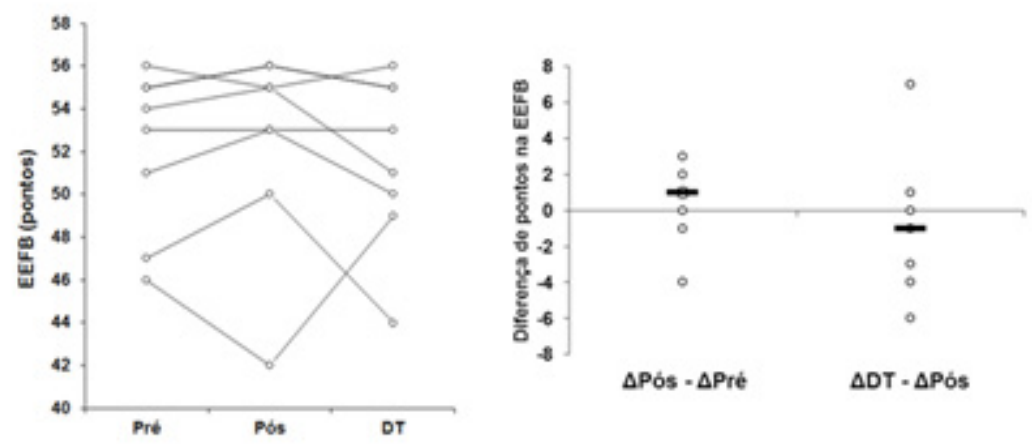

FIGURA 2 -Valores médios da EEFB, Pré (antes do programa de treinamento), Pós (após o programa de treinamento) e DT (Destreino) e diferença entre os períodos de intervenção. 

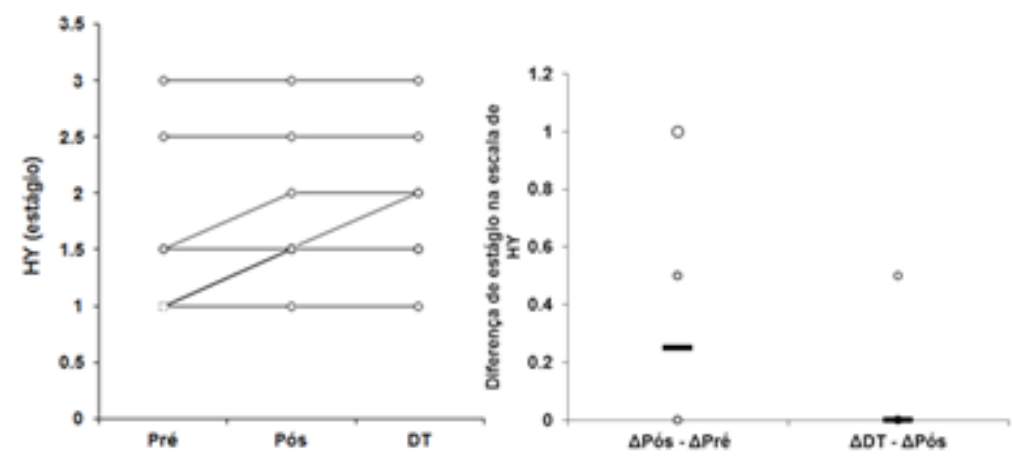

FIGURA 3 -Valores médios da escala de HY, Pré (antes do programa de treinamento), Pós (após o programa de treinamento) e DT (Destreino) e diferença entre os períodos de intervenção.

\section{Discussão}

O presente estudo teve por objetivos, realizar um programa de atividade física multimodal, e também avaliar o efeito do mesmo programa no equilíbrio dinâmico, mobilidade funcional e nas variáveis clínicas de idosos com DP idiopática. O principal achado desse estudo foi que após esses indivíduos participarem do programa multimodal, seus níveis de mobilidade funcional e equilíbrio se mantiveram.

Primeiramente em relação à mobilidade funcional avaliada por meio do TUG, observamos que o nosso programa de treinamento náo alterou esses valores em nenhum dos períodos avaliados (FIGURA 1). Há uma positiva relação entre força e a realização do teste, sendo uma combinação de flexibilidade e força, indispensáveis para a realização do TUG ${ }^{16,26}$. Como esses componentes foram trabalhados durante o treino, podemos dizer que o treino auxiliou na manutenção desses resultados. Resultados desse estudo corroboram achados da literatura que demonstraram que pacientes com DP submetidos a uma intervenção motora e exercícios multimodais não levaram a melhora em parâmetros do TUG, quando comparados a grupo controle ${ }^{27,28}$. Uma alteração mínima detectável somente seria observada se os indivíduos desse estudo apresentassem uma redução de tempo de realização do TUG em 3,5 segundos ${ }^{29}$, o que não foi observado em nenhum participante e nem tampouco em qualquer período de avaliação (FIGURA 1). Portanto, acreditamos que programas de intervenção com enfoque específico na mobilidade funcional, sejam de extrema importância para levar a manutenção no TUG, uma vez que a manutenção é um grande resultado quando nos tratamos de doenças neurodegenerativas, como é o caso da DP.

Em segundo lugar, da mesma forma se comportaram os indivíduos em relação à avaliação do equilíbrio através da EEFB, onde a manutenção com o treino foi observada nos 3 períodos de avaliação (FIGURA 2). Portanto, não houve modificações do equilíbrio, pois a alteração mínima detectável da EEFB em indivíduos com DP é de 2,84 pontos, como proposto por Lim, et al. $^{6}$. O valor obtido de EEFB também apresentou efeito teto, ou seja, pontuação máxima a ser obtida com o teste após o programa de atividade multimodal. Outros tipos de intervenção demonstraram melhora nos parâmetros do EEFB. Seja intervenção motora, exercícios multimodais, ou até mesmo intervenções físicas mais específicas (como é o caso de dança e artes marciais), podem trazer melhores resultados sobre $\mathrm{EEFB}^{27,28,30}$. Entretanto, melhoras foram observadas em intervençôes a curto prazo, e em nossa amostra a EEFB apresenta um efeito teto, dificultando a melhora nessa ferramenta.

Em relação as avaliações clínicas, apesar de não ter apresentado diferença estatística significativa, os resultados da UPDRS Total demonstraram valores diminuídos após o treino em 4,12 pontos, próximo do valor caracterizado como mínima diferença clínica importante, que relata esse valor como 4,3 pontos $^{31}$, porém, não se mantiveram assim ao avaliar o destreino. Na UPDRS III o mesmo foi observado, não havendo diferença estatisticamente 
significante. Porém, houve uma mínima diferença clinicamente importante, com a redução de 3 pontos. Segundo Shulman, et al. ${ }^{31}$, diferença de 2,7 pontos após um período de intervenção, já se caracteriza como mudança clinicamente relevante. Portanto, podemos afirmar que esses indivíduos se beneficiaram do programa de atividade física para redução dos seus sintomas motores. Na UPDRS II houve diminuição dos valores, porém não foi estatisticamente significativo, retrocedendo também após o destreino, mas mantendo seu nível de funcionalidade. Tal fato, nos leva a crer que o exercício físico, praticado de forma multimodal, por um período de 8 meses, é capaz de reduzir o avanço de alguns sintomas motores da DP. Tais resultados são similares ao de TomLinson, et al. ${ }^{30}$ que encontraram em uma meta-análise que exercício físico com características multimodais levaram a melhoras de parâmetros da UPDRS. Especulamos que essas melhoras ocorreram devido ao fato de que exercícios multimodais trabalham diversos componentes, assim como a UPDRS avalia diversos componentes motors (equilíbrio, postura, rigidez, entre outros), com isso melhoras nesses parâmetros são esperadas.

Por fim, na FIGURA 3 observamos que imediatamento após o período de intervenção, 4 participantes mudaram de estágio da DP durante esse período. Pensando dessa forma, o programa de intervenção foi benéfico, pois mesmo ocorrendo tal mudança, o equilíbrio e mobilidade funcional se mantiveram. Além disso, na TABELA 1, observamos que, apesar de não ser estatisticamente significativo, o exercício físico promoveu melhoras nos comprometimentos motores e total da DP, sendo que com a supressão do exercício, esses resultados tenderam a voltar. Como visto, o exercício induz e leva o individuo com DP a uma possível alívio dos sintomas motores da DP, porém a continuação da prática desses exercícios deve existir, pois um período de inatividade pode reverter tais e anular os benefícios do comportamento adquiridos através de exercício ${ }^{32}$.

Resultados de GobBi, et al. ${ }^{16}$ demonstraram melhora em equilíbrio e mobilidade funcional avaliados por meio das mesmas ferramentas do nosso estudo, assim como semelhança no programa de treinamento. Em questão desses resultados, os nossos demonstraram somente manutenção, podendo ser sugerido que essa manutenção ocorreu devido a nossa amostra apresentarem maiores valores de UPDRS II e III, indicando maiores comprometimentos motores e na realização das AVDs. Quanto ao destreino, HaCKNEY e EARHART $^{15}$ apresentaram melhora na EEFB em seus participantes, porém com a realização de um treinamento físico através da dança Tango com e sem parceiro. Esses resultados podem ter sido obtidos, pois por se tratar da modalidade de dança Tango, há uma série de exercícios com mudança de direção e o treinamento todo foi apenas com a dança, havendo essa aquisição na melhora de equilíbrio, decorrente de uma especificidade de treinamento.

O presente estudo apresentou algumas limitações, sendo uma dessas, a ausência de exames de imagem, onde poderia ser possível observar as alterações promovidas pelo programa de treinamento. Especula-se que o exercício físico tem atuado de maneira neuroprotetora em pacientes com DP, portanto avaliações de neuroimagens, e um desenho experimental mais adequado, facilitaria qualquer discussão sobre o papel neuroprotetor do exercício físico. Outra limitação de estudo é a falta de um grupo controle para comparar os valores obtidos com o programa de treinamento, para poder descartar a influência da progressão da doença e déficits motores, assim como, descartar os efeitos deletérios ocasionados pelo envelhecimento. Um número pequeno de participantes pode levar a uma dificuldade em generalizar os resultados desse estudo a uma população maior, representando uma outra limitação de nosso estudo. Por fim, EEFB apresenta limitaçõos devido ao seu efeito teto, e TUG pode apresentar efeitos de aprendizagem ao longo do tempo ${ }^{33}$. Portanto, ferramentas com maior acurácia clínica, como por exemplo plataforma de força para avaliar a oscilação postural ${ }^{34}$ e o uso de acelerômetros para avaliar a estabilidade postural durante o andar ${ }^{35}$, podem ser uma boa solução para avaliar o equilíbrio e a mobilidade funcional.

Mesmo com tais limitações o estudo demonstrou que o programa de exercício realizado foi eficaz para manter os resultados no equilíbrio dinâmico e mobilidade funcional, assim como foi capaz de impedir o avanço dos sintomas e do nível de acometimento da doença. Futuros estudos devem focar em intervençôes específicas as características clínicas dos pacientes com DP, e serem submetidos a uma avaliação mais acurada do equilíbrio corporal e a mobilidade funcional utilizando-se de ferramentas como plataforma de força, ou o uso de acelerômetros. 


\title{
Agradecimentos
}

À Fundação de Amparo à Pesquisa do Estado de São Paulo (2012/20498-0) e à Coordenação de Aperfeiçoamento de Pessoa de Nível Superior (BEX/2194/15-5), pelo apoio financeiro. Agradecemos também ao Programa de Atividade Física para Pacientes com Doença de Parkinson (PROPARKI) e ao Laboratório de Estudos da Postura e da Locomoçấo (LEPLO) pelo apoio logístico e intelectual.

\section{Conflito de interesse}

Os autores não apresentam nenhum conflito de interesses a declarar.

\begin{abstract}
Parkinson's disease (PD) is a neurodegenerative and progressive disease characterized by: rest tremor, rigidity, gait difficulty and postural instability. Due to that, quality of life and patient independency is compromised. Thus, different physical activity proposals have been created to be an auxiliary to medication treatment. However, many different proposals either are short-term or focused on a particular symptom. Therefore, the main aim of this study was to assess the effect of a multimodal treatment (8 months of duration) on dynamic balance and functional mobility in people with idiopathic PD. Dynamic balance was assessed by Berg Balance Scale (BBS) and functional mobility was assessed by The timed Up and Go test (TUG). Eight people with idiopathic PD participated in this study. Participants were in mild-to-moderate stage of disease (Hoehn and Yahr = 1 to 3), cognitively preserved, and were assessed on "on" phase of medication. The patients were assessed prior the multimodal treatment starts, after and 3 months after the last session (follow-up). None statistical difference was found. We can conclude that even with the progression of the disease, level of balance and functional mobility were maintained after a long-term multimodal intervention plan.
\end{abstract}

KEYwORDS: Parkinson's Disease; Dynamic Balance; Ageing; Physical Exercise; Clinical Assessment.

\section{Referências}

1. Lau LM, Breteler MM. Epidemiology of Parkinson's disease. Lancet Neurol. 2006;5:525-35.

2. Barbosa MT, Caramelli P, Maia DP, Cunningham MC, Guerra HL, Lima-Costa MF, et al. Parkinsonism and Parkinson's disease in elderly: a community-based survey in Brazil (the Bambuí Study). Mov Disord. 2006;21:800-8.

3. Hirsch MA, Farley BG. Exercise and neuroplasticity in persons living with Parkinson's disease. Eur J Phys Rehab Med. 2009; 45:215-29.

4. Petzinger GM, Fisher BE, Van Leeuwen JE, Vukovic M, Akopian G, Meshul CK, et al. Enhancing neuroplasticity in the basal ganglia: the role of exercise in Parkinson's disease. Mov Disord. 2010;25(Suppl 1):S141-5.

5. Bartels AL, Leenders KL. Parkinson's disease: the syndrome, the pathogenesis and pathophysiology. Cortex. 2009;45:91521.

6. Lim LI, Van Wegen EE, de Goede CJ, Jones D, Rochester L, Hetherington V, et al. Measuring gait and gait-related activities in Parkinson's patients own home environment: a reliability, responsiveness and feasibility study. Parkinsonism Relat Disord. 2005;11:19-24.

7. Pelicioni PHS. Impacto da doença de Parkinson sobre as características cinéticas e cinemáticas da tarefa de levantar e andar, em caidores e não caidores [dissertação]. Rio Claro (SP): Universidade Estadual Paulista, Instituto de Biociências de Rio Claro; 2014.

8. Vervoort G, Bengevoord A, Strouwen C, Bekkers EM, Heremans E, Vandenbergh W, et al. Progression of postural 
control and gait deficits in Parkinson's disease and freezing of gait: a longitudinal study. Parkinsonism Relat Disord. 2016;28:73-9. doi: 10.1016/j.parkreldis.2016.04.029.

9. Vitório R, Lirani-Silva E, Pelicioni PHS. Controle motor, ocorrência de quedas e doença de Parkinson. In: Coelho FGM, Gobbi S, Costa JLR, Gobbi LTB, organizadores. Exercício físico no envelhecimento saudável e patológico: da teoria à prática. Curitiba: CRV; 2013. p.311-23.

10. Jankovic J, Stacy M. Medical management of levodopa-associated motor complications in patients with Parkinson's disease. CNS Drugs. 2007;21:677-92.

11. Foreman KB, Addison O, Kim HS, Dibble LE. Testing balance and fall risk in persons with Parkinson's disease, an argument for ecologically valid testing. Parkinsonism Relat Disord. 2011;17:166-71.

12. Goodwin VA, Richards SH, Taylor RS, Taylor AH, Campbell JL. The effectiveness of exercise interventions for people with Parkinson's disease: a systematic review and meta-analysis. Mov Disord. 2008;23:631-40.

13. Paul SS, Canning CG, Song J, Fung VS, Sherrington C. Leg muscle power is enhanced by training in people with Parkinson's disease: a randomized controlled trial. Clin Rehabil. 2014;28:275-88.

14. Canning CG, Paul SS, Niewboer A. Prevention of falls in Parkinson's disease: a review of fall risk factors and the role of physical interventions. Neurodegener Dis Manag. 2014;4:203-21.

15. Hackney ME, Earhart GM. Effects of dance on gait and balance in Parkinson's disease: a comparison of partnered and nonpartnered dance movement. Neurorehabil Neural Repair. 2010;24:384-92.

16. Gobbi LTB, Oliveira-Ferreira MDT, Caetano MJD, Lirani-Silva E, Barbieri FA, Stella F, et al. Exercise programs improve imobility and balance in people with Parkinson's disease. Parkinsonism Relat Disord. 2009;15:S49-52.

17. Vitório R, Teixeira-Arroyo C, Lirani-Silva E, Barbieri FA, Caetano MJD, Gobbi S, et al. Effect of 6-month, multimodal exercise program on clinical and gait parameters of patients with idiopathic Parkinson's disease: a pilot study. ISRN Neurol. 2011;2011:714947.

18. Ashburn A, Fazakarley L, Ballinger C, Pickering R, McLellan LD, Fitton C. A randomised controlled trial of a home-based exercise program to reduce the risk of falling among people with Parkinson's disease. J Neurol Neurosurg Psychiatry. 2007;78:678-84.

19. Fahn S, Elton R. Members of the UPDRS.Development Comitee.The unified Parkinson's disease rating scale. In: Fahn S, Marsden CD, Calne DB, Goldstein M, editores. Recent Developments in Parkinson's disease, vol. 2. Florham Park: Mcmellam Health Care Information; 1987. p.153-164.

20. Hoehn MM, Yahr MD. Parkisonism: onset, progression and mortality. Neurology. 1967;17:427-42.

21. Miyamoto ST, Lombardi-Junior I, Berg KO, Ramos LR, Natour J. Brazilian version of the Berg balance scale. Braz J Med Biol Res. 2004;37:1411-21.

22. Pereira MP, Pelicioni PHS, Gobbi LTB. Parkinson's disease severity and motor subtype influence physical capacity components. Motriz: rev educ fís. 2013;19:605-13.

23. Orcioli-Silva D, Simieli L, Barbieri F, Rinaldi N, Vitorio R, Gobbi LTB. Effects of a multimodal exercise program on the functional capacity of Parkinson's disease patients considering disease severity and gender. Motriz: rev educ fís. 2014;20:100-6.

24. Pereira MP, Oliveira-Ferreira MDT, Caetano MJD, Vitório R, Lirani-Silva E, Barbieri FA, et al. Long-term multimodal exercise program enhances mobility of patients with Parkinson's disease. ISRN Rehabilitation. 2012;2012:1-7.

25. Falvo MJ, Schilling BK, Earhart GM. Parkinson's disease and resistive exercise: rationale, review, and recommendations. Mov Disord. 2008;23:1-11.

26. Schilling BK, Karlage RE, LeDoux MS, Pfeiffer RF, Weiss LW, Falvo MJ. Impaired leg extensor strength in individuals with Parkinson disease and relatedness to functional mobility. Parkinsonism Relat Disord. 2009;15:776-80.

27. Shen X, Wong-Yu, Mak MK. Effects of exercise on falls, balance, and gait ability in Parkinson's disease: a meta-analysis. Neurorehabil Neural Repair. 2016;30:512-27.

28. Allen NE, Sherrington C, Paul SS, Canning CG. Balance and falls in Parkinson's disease: a meta-analysis of the effect of exercise and motor training. Mov Disord. 2011;26:1605-15.

29. Huang SL, Hsieh CL, Wu RM, Tai CH, Lin CH, Lu WS. Minimal detectable change of the timed "up \& go" test and the dynamic gait index in people with Parkinson's disease. Phys Ther. 2011;91:114-21.

30. Tomlinson CL, Patel S, Meek C, Clarke CE, Stowe R, Shah L, et al. Physiotherapy versus placebo or no intervention in Parkinson's disease. Cochrane Database Syst Rev. 2012;(11):CD002817.

31. Shulman LM, Gruber-Baldini AL, Anderson KE, Fishman PS, Reich SG, Weiner WJ. The clinically important difference on the Unified Parkinson's Disease Rating Scale. Arch Neurol. 2010;67:64-70. 
32. Hirsch MA, Farley BG. Exercise and neuroplasticity in persons living with Parkinson's disease. Eur J Phys Rehabil Med. 2009;45:215-29.

33. Bloem BR, Marinus J, Almeida Q, Dibble L, Nieuwboer A, Post B, et al. Measurement instruments to assess posture, gait, and balance in Parkinson's disease: critique and recommendations. Mov Disord. 2016;31:1342-55.

34. Lahr J, Pereira MP, Pelicioni PHS, Morais LC, Gobbi LTB. Parkinson's disease patients with dominant hemibody affected by the disease rely more on vision to maintain upright postural control. Percept Mot Skills. 2015;121:923-34.

35. Brodie MA, Lovell NH, Canning CG, Menz HB, Delbaere K, Redmond SJ, et al. Gait as a biomarker? Accelerometers reveal that reduced movement quality while walking is associated with Parkinson's disease, ageing and fall risk. Conf Proc IEEE Eng Med Biol Soc. 2014;2014:5968-71.

ENDEREÇO DE CORRESPONDÊNCIA:

Paulo Henrique Silva Pelicioni

Neuroscience Research Australia,

Barker Street, Randwick, 2031

Sydney - AUSTRALIA

E-mail: p.pelicioni@neura.edu.au
Submetido: $15 / 05 / 2016$

1a revisão: 30/11/2016

2a revisão: 16/03/2017

Aceito: 08/05/2017 